\title{
HIGH-FIELD ACCELERATOR MAGNETS BEYOND LHC
}

\author{
A. Devred \\ CEA/Saclay, Gif-sur-Yvette, France \& CERN, Accelerator Technology Division, Geneva, Switzerland
}

The LHC magnet R\&D Program has shown that the limit of NbTi technology at $1.8 \mathrm{~K}$ was in the range 10 to $10.5 \mathrm{~T}$. Hence, to go beyond the 10 - T threshold, it is necessary to change of superconducting material. Given the state of the art in HTS, the only serious candidate is Nb3Sn. A series of dipole magnet models built at Twente University and LBNL and a vigorous program underway at FNAL have demonstrated the feasibility of $\mathrm{Nb} 3 \mathrm{Sn}$ magnet technology. The next step is to bring this technology to maturity, which requires further conductor and conductor insulation development and a simplification of manufacturing processes. After outlining a roadmap to address outstanding issues, we evoke the US proposal for a second generation of LHC Insertion Region (IR) magnets and the Next European Dipole (NED) initiative promoted by the European Steering Group on Accelerator R\&D (ESGARD). 


\title{
HIGH-FIELD ACCELERATOR MAGNETS BEYOND LHC
}

\author{
A. Devred, ${ }^{*}$ CEA/Saclay, DSM/DAPNIA/SACM, 91191 Gif-sur-Yvette, France \\ \& CERN, Accelerator Technology Division, CH-1211 Geneva, 23, Switzerland
}

Abstract

The LHC magnet R\&D Program has shown that the limit of $\mathrm{NbTi}$ technology at $1.8 \mathrm{~K}$ was in the range 10 to $10.5 \mathrm{~T}$. Hence, to go beyond the 10-T threshold, it is necessary to change of superconducting material. Given the state of the art in HTS, the only serious candidate is $\mathrm{Nb}_{3} \mathrm{Sn}$. A series of dipole magnet models built at Twente University and LBNL and a vigorous program underway at FNAL have demonstrated the feasibility of $\mathrm{Nb}_{3} \mathrm{Sn}$ magnet technology. The next step is to bring this technology to maturity, which requires further conductor and conductor insulation development and a simplification of manufacturing processes. After outlining a roadmap to address outstanding issues, we evoke the US proposal for a second generation of LHC Insertion Region (IR) magnets and the Next European Dipole (NED) initiative promoted by the European Steering Group on Accelerator R\&D (ESGARD).

\section{WHY DO WE NEED HIGHER-FIELD ACCELERATOR MAGNETS?}

\section{The Push Towards Higher Fields}

For a given tunnel size, the energy of a circular machine is limited by the strength of bending magnets. Moreover, for both linear and circular colliders, the luminosity is determined (mainly) by the optics of Interaction Regions (IR's), which is itself limited by the strength and quality of IR magnets. Over the years, there has been a constant push from the High-Energy Physics (HEP) community to keep developing higher-field and higher-field gradient accelerator magnets.

\section{Brief History}

The push towards higher fields led naturally to the use of superconductors. Worthy of mention is the pioneer work carried out by W.B. Sampson at Brookhaven National Laboratory (BNL) in the mid 1960's, illustrated in Figure 1 by a $76-\mathrm{mm}$-aperture, $85-\mathrm{T} / \mathrm{m}$ quadrupole magnet model wound from $\mathrm{Nb}_{3} \mathrm{Sn}$ ribbons and cold tested in January 1966 [1]. (Note that the aperture and field gradient of this model are similar to those of the HERA quadrupole magnets developed 15 years later [2].)

The first successful use of superconducting magnets in a machine took place at the Tevatron, at Fermi National Accelerator Laboratory (FNAL) [3]. The Tevatron, which relies on 774 6.1-m-long, 76.2-mm-aperture, 4-T arc dipole magnets, was commissioned in 1983 and has been running very reliably since then. It was instrumental in demonstrating the feasibility and reliability of superconducting magnet systems and has paved the way to their commercial applications (such as Magnetic Resonance Imaging or MRI systems).

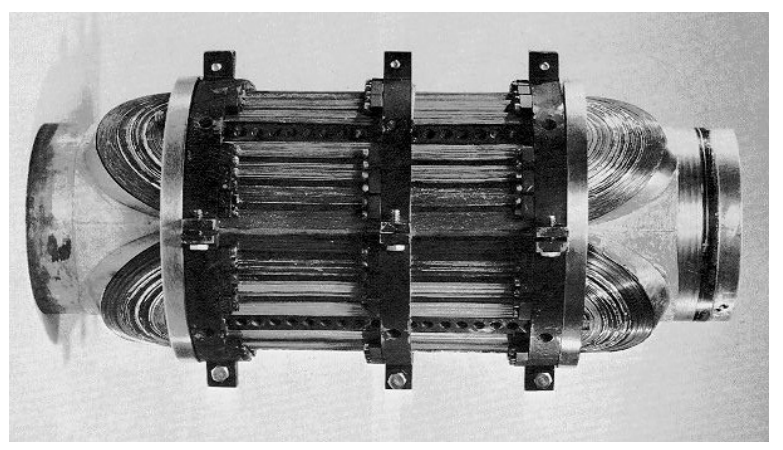

Figure 1: 76-mm-aperture, $85-\mathrm{T} / \mathrm{m}$ quadrupole model wound from $\mathrm{Nb}_{3} \mathrm{Sn}$ ribbons by W.B. Sampson at BNL in 1965 [1].

Since the time of the Tevatron, significant progress has been made in the design and production of superconductors and accelerator magnets, leading to a gain of a factor $\sim 2$ in dipole field. The ongoing superconducting magnet productions for the Large Hadron Collider (LHC) at CERN, which, among others, call for 1232 14.2-m-long, 56-mm-twin-aperture, 8.33-T arc dipole magnets, is the culmination of 20 years of superconducting accelerator magnet development around the world [4]. The idea of building the LHC first emerged in 1982 [5], and the machine is expected to be turned on in the Spring of 2007, a mere 25 years later.

\section{What's next?}

In addition to arc dipole and quadrupole magnets, LHC also requires a number of superconducting IR magnets, including triplets of final-focusing quadrupole magnets, which are presently being built at FNAL and KEK [6]. Due to the high radiation doses to which they will be subjected, the life expectancy of these magnets is estimated around 7 years. Hence, it is likely that they will have to be replaced in 2015 , thereby offering the opportunity of upgrading LHC IR optics to improve luminosity.

Several scenarios of LHC IR upgrades are already being considered [7], [8]. The most conservative ones keep the present optics layout but rely on stronger finalfocusing quadrupole magnets. The most innovative ones call for a different optics layout, where the beamseparation dipole magnets are located in front of the final-focusing quadrupole magnets to reduce long-range, beam-beam interactions, as illustrated in Figure 2. In any case, these various scenarios require the development of large-aperture, high-field or high-field-gradient magnets.

Mid 2010's is also the earliest time frame when one can expect to need final-focusing quadrupole magnets for any of the proposed linear collider projects. In the case of linear colliders, the magnet requirements are very IRdesign dependent. 


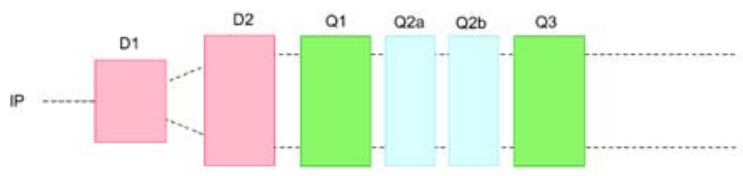

Figure 2: LHC-IR upgrade scenario where the beam-separation dipoles (D1 and D2) are located in front of the inner-triplet of final-focusing quadrupoles (Q1, Q2 and Q3) [7].

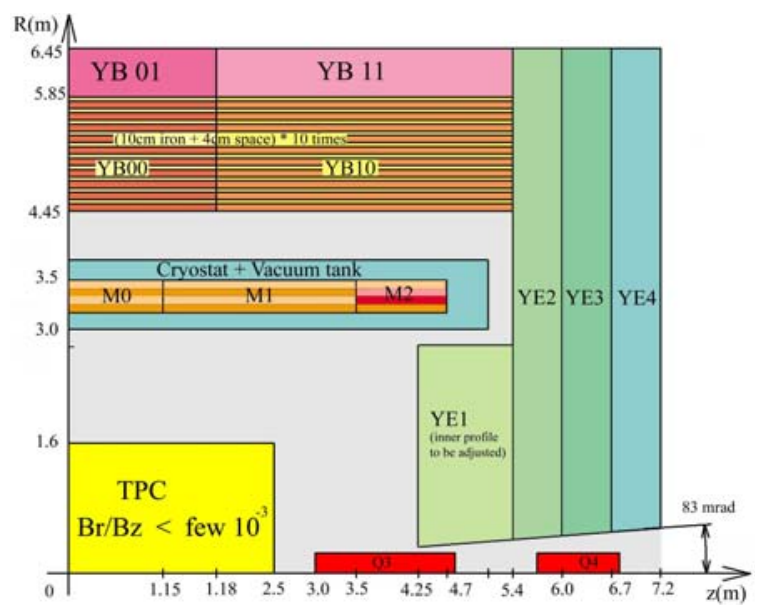

Figure 3: Layout of TESLA $1^{\text {st }}$ IR where the final-focusing quadrupoles (Q3 \& Q4) are located inside the detector solenoid (whose winding sections are labelled M0, M1 \& M2) [9].

For the first IR of the Tera Electron volts Superconducting Linear Accelerator (TESLA), the layout proposed in the Technical Design Report (TDR) relies on final-focusing quadrupole magnets producing $250 \mathrm{~T} / \mathrm{m}$ in a 56-mm-single-aperture. However, these magnets are positioned very close to the interaction point and must operate in the 4-T background field of the detector solenoid (see Figure 3) [9]. For the Next Linear Collider (NLC), or the second IR of TESLA, where it is foreseen that the two beams cross with a large angle, the finalfocusing quadrupole magnets must be made very compact (i.e., with a small overall outer radius) so as to clear the way for the crossing beam [10].

\section{Roadmap for High-Field Magnet R\&D}

Given the prospects outlined above, a roadmap for high-field accelerator magnet development appears to be

- $\quad$ To get ready for LHC IR upgrade in 2015 (which calls for large-aperture, high-performance dipole or quadrupole magnets; note that here cost is not the primary issue),

- $\quad$ To develop final-focusing quadrupole magnets for implementation in a linear collider IR in the mid2010's (which calls for LHC-type quadrupole magnets in a solenoidal background field or for compact quadrupole magnets; note that here also cost is not the primary issue),

- To promote generic magnet R\&D aimed at LHC energy upgrade or a VLHC in the mid 2020's (which calls for high-performance, low-cost dipole and quadrupole magnets).

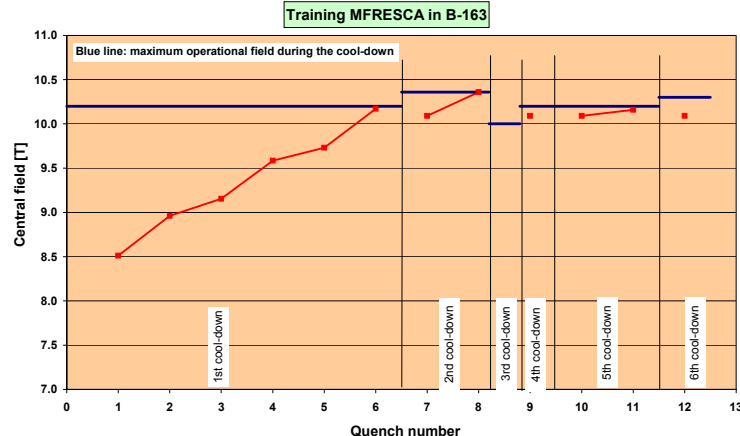

Figure 4: Quench performance of 88-mm-aperture (NbTi) MFRESCA dipole magnet model at CERN [13].

\section{WHY IS IT SO HARD?}

Twelve years may seem like a long time to develop a new dipole or quadrupole magnet design for LHC or linear collider IR applications. The issue, however, is that we cannot extrapolate existing designs and that we need to change of superconductor technology.

\section{State of the Art in NbTi}

Since the time of the Tevatron, the most widely used superconductor is a ductile alloy of niobium-titanium (NbTi) easy to co-process with copper by conventional extrusion and drawing techniques [11]. The world production of $\mathrm{NbTi}$ is estimated around 1500 metric tons per year, mainly under the form of multifilamentary composite wires for use in MRI magnets.

After several iterations, the CERN/LHC dipole magnet R\&D program was successful in working out a design suitable for industrial production, but it demonstrated also that the limit of $\mathrm{NbTi}$ magnets (cooled down to superfluid helium at $1.8 \mathrm{~K}$ ) lied in the 10-to-10.5-T range. This is illustrated in Figure 4, which shows the quench performance of the 88-mm-single-aperture MFRESCA dipole magnet, designed and built by a team led by D. Leroy and presently implemented in the superconducting cable test facility at CERN [12], [13]. Hence, to go beyond the present limitations and cross the 10-T threshold, it appears necessary to change of superconducting material.

\section{Beyond $\mathrm{NbTi}: \mathrm{Nb}_{3} \mathrm{Sn}$}

High Temperature Superconductors (HTS) are not yet ready for large-scale applications requiring high current densities under high magnetic fields, and it is likely that it will take at least another decade before they become competitive. The limited gain that can be expected from ternary NbTiTa alloys does not seem worth the investment. The present upper critical field of $\mathrm{MgB}_{2}$ wires is two low. $\mathrm{Nb}_{3} \mathrm{Al}$ exhibits promising properties, but there are serious manufacturing issues that have yet to be resolved. It follows that the only serious candidate to succeed $\mathrm{NbTi}$ is the intermetallic compound $\mathrm{Nb}_{3} \mathrm{Sn}$, whose world production is estimated around 15 metric tons per year (also under the form of multifilamentary composite wires) [14]. 

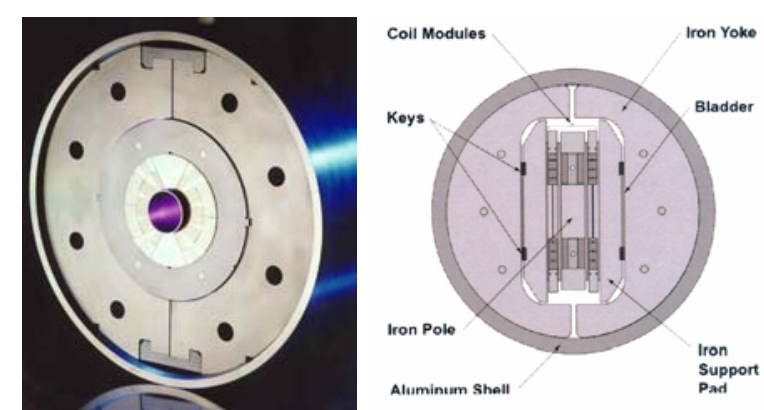

Figure 5: Record-breaking $\mathrm{Nb}_{3} \mathrm{Sn}$ dipole magnet models; (a) 50 -aperture, $\cos \theta$-type MSUT at Twente University (left) [19] and (b) 25-mm-gap, racetrack-type RD-3 at LBNL (right) [21].

$\mathrm{Nb}_{3} \mathrm{Sn}$ has a critical temperature, $T_{\mathrm{C}}$, and an upper critical field, $B_{\mathrm{C} 2}$, that are about twice those of $\mathrm{NbTi}$. However, once formed, it becomes brittle and its critical parameters $\left(T_{\mathrm{C}}, B_{\mathrm{C} 2}\right.$, and the critical current density, $\left.J_{\mathrm{C}}\right)$ are strain sensitive [15]. The brittleness and strainsensitivity of $\mathrm{Nb}_{3} \mathrm{Sn}$ require a different approach to all manufacturing processes and, so far, have limited its use to specific applications (such as insert coils for high-field Nuclear Magnetic Resonance or NMR spectrometers).

\section{Progress on $\mathrm{Nb}_{3} \mathrm{Sn}$ Technology}

In spite of the aforementioned difficulties, significant progress has been made over the last decade thanks to

- The successful manufacturing and tests of the model coils for the International Thermonuclear Experimental Reactor (ITER) project, which, among other, have required the production of $\sim 30$ metric tons of $\mathrm{Nb}_{3} \mathrm{Sn}$ wires [16], [17],

- A US National Program for the development of high-performance $\mathrm{Nb}_{3} \mathrm{Sn}$ wires, supervised by R.M. Scanlan at Lawrence Berkeley National Laboratory (LBNL), which has led already to a three-to-four-fold increase in $J_{\mathrm{C}}$ with respect to ITER model coil specifications [18],

- A series of record-breaking dipole magnet models, opening the 10-to- $15 \mathrm{~T}$ range, including the 50 $\mathrm{mm}$-aperture, $\cos \theta$-type, MSUT model, built at Twente University and cold tested at CERN in 1995 , which reached $11 \mathrm{~T}$ on its first quench at $4.4 \mathrm{~K}$ (Fig. 5(a)) [19], the 50-mm-aperture, $\cos \theta$-type, D2 0 model, built and cold tested at LBNL, which, after some training, reached $13.5 \mathrm{~T}$ at $1.8 \mathrm{~K}$ in 1997 [20], and the 25-mm-gap, racetrack-type, RD-3 model, also built and cold tested at LBNL, which, after some training reached $14.7 \mathrm{~T}$ at $4.2 \mathrm{~K}$ in 2001 (Fig. 5(b)) [21].

This progress shows that, although the $\mathrm{Nb}_{3} \mathrm{Sn}$ technology is not yet mature, it could be at hand for the high-field and high-field-gradient accelerator magnets needed for LHC IR upgrade and for the IR's of future linear colliders. However, it is clear also that we need to keep working hard if we want to turn these few successful demonstrators into accelerator-class devices that can be implemented in a machine within 10 to 15 years.

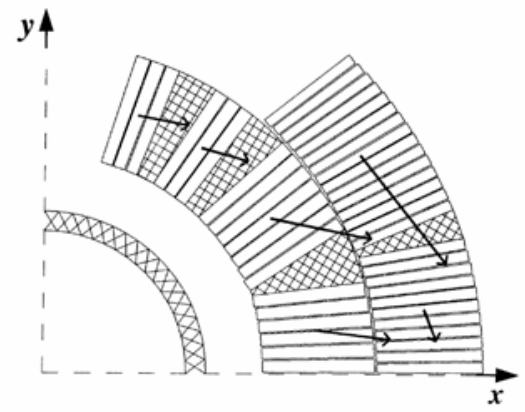

Figure 6: Lorentz force distribution in a quadrant of a $\cos \theta$ dipole magnet coil assembly (Courtesy R. Gupta).

\section{WHAT DO WE HAVE TO DO?}

\section{Task List}

Given the present state of the art on accelerator magnet technology and the requirements foreseen for LHC IR upgrade and for IR's of future linear colliders, we need

- To revisit magnetic and mechanical designs to achieve enhanced performances with magnet coils made from brittle materials,

- To address coil cooling issues under high beam losses,

- To keep promoting high-performance $\mathrm{Nb}_{3} \mathrm{Sn}$ wire development (and to ensure the survival of multiple suppliers around the world),

- To improve robustness and assess radiation hardness of $\mathrm{Nb}_{3} \mathrm{Sn}$ conductor insulation (see, for instance, the innovative insulation scheme developed by Composite Technology Development, Inc., or CEA/Saclay [22]),

- To put into practice all of the above in magnet models and prototypes.

Of course, a number of laboratories around the world are already actively tackling these issues, including BNL, FNAL and LBNL in the USA, and CEA/Saclay and Twente University in Europe. A detailed review of the ongoing programs can be found elsewhere [23]. Given the limited space at our disposal, let us single out the problem of magnetic design.

\section{Revisiting Magnetic Design}

Most superconducting accelerator magnets rely on socalled saddle-shape coils, which, in their long straight sections approximate $\cos \theta$ or $\cos 2 \theta$ conductor distributions. Such designs were first optimized at BNL in the mid 1960's using R.A. Beth's complex formalism [24]. They are very efficient in terms of superconductor use and to control field quality, but, as illustrated in Figure 6, they result in a transverse stress accumulation towards the coil assembly midplane that could become detrimental when dealing with brittle conductors. Nevertheless, and in spite of the very high Lorentz forces developed in the MSUT and D20 models (which were both of $\cos \theta$-type), the performance of these magnets did not appear to be limited by stress-induced degradation. 


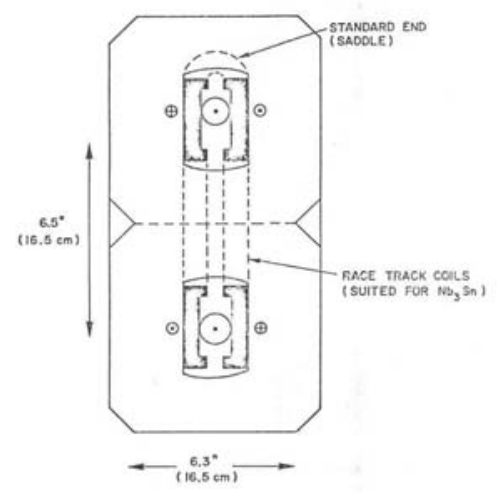

Figure 7: Original design of dual-bore dipole magnet relying on Racetrack-type coils first proposed by G.K. Danby (BNL) in 1983 [25]

The good results of the MSUT and D20 models indicate that we have not yet reached a hard limit on the mechanical point of view. This implies that, for LHC IR upgrade and for the first IR of TESLA, we can still safely rely on "conventional" $\cos \theta$ or $\cos 2 \theta$ designs.

However, in the longer run, and given the very open time-scale for a LHC energy upgrade or a VLHC, it is, of course, worthwhile to investigate other designs. Among possible candidates, let us mention the racetrack-type coil design, illustrated in Figure 7, which was first proposed by G. Danby at BNL in 1983 [25] and was subsequently resuscitated by R. Gupta in 1996 [26]. This design has become the workhorse of the LBNL high-field magnet program and was used for the RD-3 model.

As a curiosity, let us also mention the double-helix coil design, illustrated in Figure 8 [27], which was investigated in the early 1970's and which is also being brought back into actuality by several authors.

\section{HOW TO GET ORGANIZED?}

At present, most of the worldwide resources are (for good reasons) used up by LHC and very little is left for accelerator magnet R\&D. Given the little resources that are available

- We cannot afford to do everything at once, and we need to target our activities towards a limited number of clearly identified goals,

- We should avoid unnecessary work duplication and try to coordinate efforts among interested partners.

Some attempts at developing integrated programs are presently being made both in the USA and in the EU.

\section{US LARP}

BNL, FNAL and LBNL are presently collaborating to the US-LHC Accelerator Project, which, among others, include the in-kind contribution of a number of superconducting (NbTi) LHC IR magnets. In parallel, all 3 laboratories are also pursuing independent high-field magnet programs that are well described in the literature.

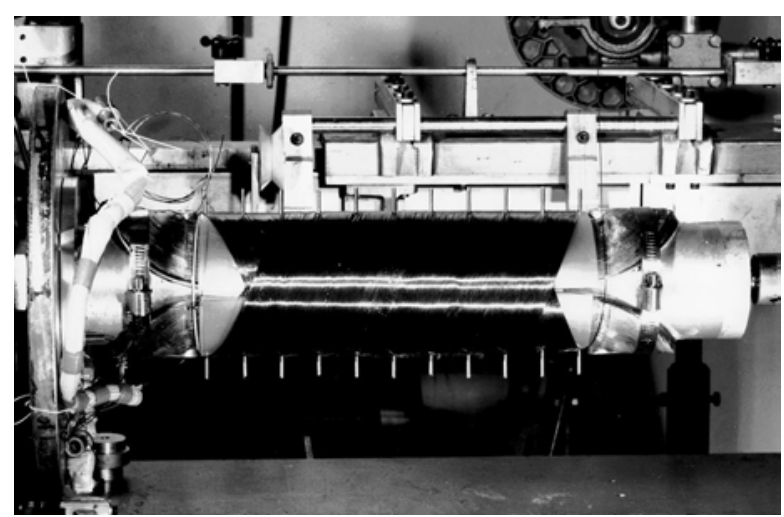

Figure 8: Dipole magnet model based on a double helix coil under manufacturing at CEA/Saclay in 1974 [27].

The US-LHC Accelerator Project team, led by J. Strait, FNAL, is now proposing to extend the present collaboration beyond LHC construction and is developing a US-LHC Accelerator Research Program (LARP) aimed at LHC IR upgrade. The Program scope and details are still under discussion. It will include $\mathrm{Nb}_{3} \mathrm{Sn}$ magnet $\mathrm{R} \& \mathrm{D}$ work on both dipole and quadrupole magnets, but will focus mainly on large-aperture (up to $110 \mathrm{~mm}$ ), high-field-gradient $(>200 \mathrm{~T} / \mathrm{m})$ quadrupole magnets [28].

\section{EU CARE/NED Proposal}

In October 2002, the European Committee for Future Accelerators (ECFA) has set up the European Steering Group for Accelerator R\&D (ESGARD), chaired by R. Aleksan, CEA/Saclay, with the mandate of preparing a coherent set of bids to apply for EU funding [29]. The first outcome of ESGARD is the Coordinated Accelerator Research in Europe (CARE) proposal of Integrated Activities (IA), which was submitted to the EU on April 15, 2003.

The CARE proposal is a first attempt at integrating all HEP-related accelerator R\&D in Europe and is supported by more than 100 institutes. It includes 3 Network Activities (linear colliders, neutrino beams and hadrom colliders) and 6 Joint Research Activies (JRA's), to develop specific hardware pieces or systems. One of the JRA's, nicknamed NED (for Next European Dipole) focuses on high field magnets.

The main objective of the NED JRA is to develop a large-aperture (up to $88 \mathrm{~mm}$ ), high-field (up to $15 \mathrm{~T}$ ) dipole magnet model, relying on high performance $\mathrm{Nb}_{3} \mathrm{Sn}$ conductors (non- $\mathrm{Cu} J_{\mathrm{C}}$ up to $1500 \mathrm{~A} / \mathrm{mm}^{2}$ at $4.2 \mathrm{~K}$ and $15 \mathrm{~T}$ ). Such magnet is aimed at demonstrating the feasibility of the LHC IR upgrade scenario illustrated in Figure 2 where the beam-separation dipole magnets are located in front of the final-focusing quadrupole magnets, and it complements the US LARP. In addition, the NED magnet could be used to replace the MFRESCA magnet and upgrade the CERN cable test facility. 
The NED JRA involves 7 collaborators (CEA/Saclay, CERN, INFN Milan and Genoa, RAL, Twente University and Wroclaw University) plus several industrial partners. The EU decision is expected before the end of the year. If approved, the program will start on January $1^{\text {st }}, 2004$, and the magnet should be cold tested in the Fall of 2008.

\section{CONCLUSION}

The US LARP and the EU NED proposal offer unique opportunities to develop the next generation of high-field magnets that will be needed for LHC-IR upgrade and for the IR's of future linear colliders.

Beyond HEP applications, such programs will help superconducting wire manufacturers to keep improving the performance and quality of their commercial $\mathrm{Nb}_{3} \mathrm{Sn}$ products (such as high-field NMR wires).

Furthermore, lessons learned from $\mathrm{Nb}_{3} \mathrm{Sn}$ should also help future HTS applications.

Let us hope that these two programs will be funded at a suitable level and that the accelerator magnet community will be given the means of maintaining its level of Excellency and of preparing its future...

\section{REFERENCES}

[1] W.B. Sampson, "Superconducting Magnets for Beam Handling and Accelerators." In H. Hadley (ed.), Proc. of $2^{\text {nd }}$ Int. Conf. on Magnet Technology, pp. 574-578, 1967.

[2] R. Auzolle, A. Patoux, et al., "Construction and test of superconducting quadrupole prototypes for HERA," Journal de Physique, Vol. 45 (Colloque C1, supplément au No. 1), pp. 263-266, 1984.

[3] H.T. Edwards, "The Tevatron Energy Doubler: a Superconducting Accelerator," Ann. Rev. Nucl. Part. Sci., Vol. 35, pp. 605-660, 1985.

[4] L. Rossi, "LHC Superconducting Magnets," in these Proceedings.

[5] H. Schopper, "LEP and Future Options" Proc. of $12^{\text {th }}$ Int. Conf. on High Energy Accel., pp. 658-663, 1983.

[6] J.S. Kirby, "Production Status of the LHC Inner Triplet Magnet System," to appear in the Proceedings of the Applied Superconductivity Conference, Houston, TX, August 4-9, 2002.

[7] O. Brüning, R. Cappi, et al., "LHC Luminosity and Energy Upgrade: A Feasibility Study," CERN LHC-Project Report 626, December 1, 2002.

[8] J. Strait, M. Lamm, et al., "Towards a New LHC Interaction Region Design for a Luminosity Upgrade," in these Proceedings.

[9] A. Devred, C. Gourdin, et al., "Conceptual Design for the Final Focus Quadrupole Magnets for TESLA," DESY TESLA-2001-17, CEA/DSM DAPNIA-STCM-01-03, 2001.

[10] B. Parker, BNL, private communication, 2003.

[11] P.J. Lee, D.C. Larbalestier, et al., "Chapter 5: Fabrications Methods." In K. Osamura (ed.), Composite Superconductors, New York, NY: Marcel Dekker, Inc., pp. 237-321, 1994.
[12] D. Leroy, G. Spigo, et al., "Design and Manufacture of a Large-Bore $10 \mathrm{~T}$ Superconducting Dipole for the CERN Cable Test Facility," IEEE Trans. Appl. Supercond., Vol. 10 No. 1, pp. 178-182, 2000.

[13] A.P. Verweij, CERN, private communication, 2003.

[14] P.J. Lee, "Advances in Superconducting Strands for Accelerator Magnet Application," in these Proceedings.

[15] J.W. Ekin, "Strain Effects in Superconducting Compounds" Adv. Cryo. Eng. (Materials), Vol. 30, pp. 823-836, 1984.

[16] N. Martovetsky, P. Michael, et al., "ITER CS Model Coil and CS Insert Test Results," IEEE Trans. Appl. Supercond., Vol. 11 No. 1, pp. 2030-2033, 2001.

[17] R. Heller, D. Ciazynski, "Evaluation of the Current Sharing Temperature of the ITER Toroidal Field Model Coil," to appear in the Proceedings of the Applied Superconductivity Conference, Houston, TX, August 4-9, 2002.

[18] R.M. Scanlan and D.R. Dietderich, "Progress and Plans for the US HEP Conductor Development Program" to appear in the Proceedings of the Applied Superconductivity Conference, Houston, TX, August 4-9, 2002.

[19] A. den Ouden, H. ten Kate, et al., "Quench characteristics of the $11 \mathrm{~T} \mathrm{Nb}_{3} \mathrm{Sn}$ model dipole magnet MSUT," Proc. of $15^{\text {th }}$ Int. Conf. on Magnet Technology, Beijing, China: Science Press, pp. 339-342, 1998.

[20] A.D. McInturff, R. Benjegerdes, et al., "Test Results for a High Field (13T) $\mathrm{Nb}_{3}$ Sn Dipole," Proc. of 1997 Part. Accel. Conf., pp. 3212-3214, 1998.

[21] R. Benjegerdes, P.Bish, et al., "Fabrication and Test Results of a High Field, $\mathrm{Nb}_{3} \mathrm{Sn}$ Superconducting Racetrack Dipole Magnet," Proc. of 2001 Part. Accel. Conf., pp. 208-210, 2001.

[22] A. Devred, "Insulation Systems for $\mathrm{Nb}_{3} \mathrm{Sn}$ Accelerator Magnet Coils Manufactured by the Wind \& React Technique," IEEE Trans. Appl. Supercond., Vol. 12 No. 1, pp. 1232-1237, 2002.

[23] M.J. Lamm, " $\mathrm{Nb}_{3} \mathrm{Sn}$ Accelerator Magnet Development Around the World," to appear in the Proceedings of the Applied Superconductivity Conference, Houston, TX, August 4-9, 2002.

[24] R.A. Beth, "Complex Representation and Computation of Two-Dimensional Magnetic Fields," J. Appl. Phys., Vol. 37 No. 7, pp. 2568-2571, 1966.

[25] G. Danby, R. Palmer, et al., "Panel Discussion of Magnets for a Big Machine," Proc. of $12^{\text {th }}$ Int. Conf. on HighEnergy Accel., pp.52-62, 1983.

[26] R.C. Gupta, "A Common Coil Design for High Field 2-in1 Accelerator Magnets," Proc. of 1997 Part. Accel. Conf., pp. 3344-3346, 1998.

[27] J. LeBars, private communication, 1974

[28] A.V. Zlobin, V.V. Kashikin, et al., "Aperture Limitations for $2^{\text {nd }}$ Generation $\mathrm{Nb}_{3} \mathrm{Sn}$ LHC IR Quadrupoles," in these Proceedings.

[29] http://esgard.lal.in2p3.fr 